\title{
Myocardial Perfusion by Contrast Echocardiography. Establishment of Normal Pattern of Intracoronary Injection and Safety in Humans
}

\author{
Fernando Morcerf, Armando Luiz Cantisano, Flavia Salek, Carlos Alberto Mendonça, Guilherme \\ Paulino, Flavio Cure Palheiro, Antonio Carlos Nogueira, Wanderley Quarte Pereira, Álvaro Moraes
}

Rio de Janeiro, RJ - Brazil

\begin{abstract}
Objective - To establish the normal pattern and safety of echocardiographic contrast in patients with no significant obstruction of epicardial coronary arteries.

Methods - 67 patients with normal coronary arteries or obstructions $<50 \%$ were selected from 277 patients who underwent coronary angiography $(C A)$. Mean age was $56 \pm 11$ years and 36 were males. At the end $C A$, echocardiographic contrast was selectively injected into each coronary artery. The parasternal short axis of the left ventricle $(L V)$ was divided into six segments: anterior $(A)$, antero-lateral $(A L)$, postero-lateral $(P L)$, posterior $(P)$, infero-septal (IS) and antero-septal (AS). Anterolateral (ALPM) and posteromedial papillary muscles (PMPM) were also considered. The pattern and intensity of the appearance of the myocardial contrast was visually analyzed.

Results - The right coronary artery (RCA) was dominant in 60 patients. Contrast appearance was sudden and simultaneous in the 3 muscle layers. All segments could be contrasted after the injection in both coronary arteries. $100 \%$ of the AS, A and AL segments, 97\% of the PL and $98 \%$ of the ALPM were perfused by the left coronary artery (LCA). P and IS segments were perfused by the RCA in $85 \%$ and $82 \%$, respectively, and by a dominant LCA in $71 \%$ of the cases. The PMPM was perfused by a dominant RCA in $77 \%$ and by a dominant LCA in $86 \%$. There were no symptoms.
\end{abstract}

Conclusion - Intracoronary injection of the sonicated solution is a safe procedure that allows for an excellent opacification of the myocardium and can potentially be used during routine $C A$.

Keywords: myocardial perfusion, echocardiography, myocardial contrast echocardiography, microbubbles

Pró-Cardíaco Hospital and ECOR-Centro de Diagnóstico Cardiovascular - Rio de Janeiro

Mailing address: Fernando Morcerf - R Jardim Botânico 468 - 22461-000 Rio de Janeiro, RJ - Brazil
Contrast echocardiography using sonicated agents (microbubbles) injected into the coronary arteries during coronary angiography is a new technique that can be used to demonstrate perfusion in the coronary artery territory ${ }^{1-6}$ and therefore, to detect perfusion abnormalities due to coronary artery obstructions ${ }^{7-12}$.

Although there are several studies on the use of this technique to evaluate myocardium at risk ${ }^{2,13-14}$, myocardial viability ${ }^{15-18}$, presence of collaterals ${ }^{19-21}$, the results of angioplasty ${ }^{22,23}$, and of myocardial revascularization surgeries ${ }^{24}$, few studies have been performed to define the normal pattern of the appearance and distribution of contrast in humans without significant obstruction of the epicardial arteries. As far as we know from the literature reviewed at the time of the present study, studies performed to establish the normal perfusion pattern and the safety of this technique involved only a small number of patients. Feinstein et al ${ }^{25}$ studied 14 patients to establish the normal pattern of the method and Moore et $\mathrm{al}^{26}$ studied 10 patients to determine its safety. It is evident that both studies are limited in their conclusion due to the small number of patients.

Therefore, the purpose of this study was to determine what the normal pattern of myocardial perfusion was when analyzed by echocardiogaphic contrast with microbubbles, as well as its safety in a significant number of patients who underwent routine coronary angiography to evaluate chest pain.

\section{Methods}

Three hundred and ten contrast echocardiograms with microbubbles were performed in 277 nonconsecutive patients who underwent coronary angiography to investigate chest pain. Sixty-seven patients without valvar, myocardial, pericardial or hypertensive disease and who also had normal coronary arteries or less than a $50 \%$ obstruction by 
angiography were selected if they had a technically adequate contrast echocardiogram, where all LV walls could be well visualized after contrast injection.

Age ranged from 36 to 88 years (mean $56 \pm 11$ years) and 36 were males. The study was approved by the ethics Committee of Pró-Cardíaco Hospital in Rio de Janeiro, and oral consent was obtained from each patient.

Coronary angiography was performed in all patients through the right brachial artery, using Sones's technique. At the end of the coronary angiography and the left ventriculography, echocardiogarphic contrast was selectively injected into each coronary artery. Continuous twodimensional echocardiography (2DE) images were obtained, beginning two minutes before injection of the echocardiographic contrast, so that adequate control of the 2DE image could be obtained and then kept constant throughout the exam. The procedure was interrupted only after total disappearance of the contrast.

2DE was performed using commercially available equipment (Aloka SSD-720), with a mechanical sectorial 3.5 $\mathrm{MHz}$ transducer. The exams were performed with the patient lying in the supine position. Images were obtained from the left parasternal window and the short axis of the left ventricle at the level of the papillary muscles was continuously recorded on videotape (VHS system) to be analyzed afterwards.

The echcocardiogaphic contrast was obtained by sonification of the contrast that is used for routine angiographic exams in the Hemodynamic Department of the PróCardíaco Hospital (Pyelograph-76). It was prepared in the following standardized fashion consisting of: 1) $15 \mathrm{ml}$ of Pyelograph-76 were placed in a $20 \mathrm{ml}$ plastic syringe with the horner of the sonicator located at least $1 \mathrm{~cm}$ inside the contrast. 2) The sonicator (XL 2020, Heart Systems Inc, Farmingdale, NY, USA), with a $20 \mathrm{KHz}$ was turned on, operating at its maximum energy. Then, the syringe was raised and lowered three to four times in order to obtain an adequated mixture. 3) This solution was injected after two minutes of its preparation.

Coronary injections of the echocardiographic contrast were manually performed as a routine procedure of the coronary angiography, with the catheter positioned inside the coronary artery at its ostium. Two ml of the solution were injected, followed by a washing out with 4 to $7 \mathrm{ml}$ of saline. If contrast could not be adequately seen in the LV walls or was detected in large amounts within the ventricular cavity, the position of the catheter was checked, repositioned and, if necessary, another injection was performed. The same technique was used if the 2DE images obtained were considered inadequate. Patients' symptoms were monitored throughout the injection period and an electrocardiogram (EKG) was continuously registered.

Coronary angiography was interpreted by two cardiologists using the visual criteria to quantify coronary artery obstructions. A coronary artery was considered dominant when it gave rise to the posterior descending artery.

The effect of the echocardiographic contrast was analyzed by two independent observers, who reviewed the recorded 2DE images without knowing the results of the coronary angiogaphy beforehand. The short axis of the LV was divided into 6 segments: anterior, antero-lateral, postero-lateral, posterior, infero-septal, antero-septal, plus anterolateral and posteromedial papillary muscles (figure 1). The pattern of the contrast appearance was visually analyzed. A segment was considered to be perfused by a certain coronary artery when, after injection into that artery, more than $50 \%$ of the area of that segment became contrasted. Consensus between the two observers was obtained to determine contrast distribution in the segments, both transmurally and intersegmentally. No segment that was not adequately seen was analyzed.

\section{Results}

The number of injections to obtain standard contrast varied from 2 to 4 per patient. Myocardial contrast was visually considered to have the same intensity, independent of the coronary artery where it was injected. Contrast appearance was characterized by sudden and simultaneous visualization of the three layers (endo-, meso- and epicardium) of the muscle wall of the segment which was perfused by the coronary artery that was being studied (figure 2). There was no delay in the appearance of the contrast, nor any visual difference in its intensity among segments that were perfused by the same coronary artery. Limits between contrasted and noncontrasted segments were always well delineated. Time between contrast appearance in the myocardium and its disappearance ranged from 20 to $90 \mathrm{~s}$ (mean value $=50.8 \pm 19.9 \mathrm{~s}$ ). There was great variablity in time in all studied patients, even when the injection was repeated in the same coronary artery of the same patient.

Right coronary artery (RCA) was considered dominant by perfusion in 60 patients and the left coronary artery in only 7. Contrast distribution among segments was dependent on the coronary artery that was infused and on the pattern of coronary dominance (figure 3 ).

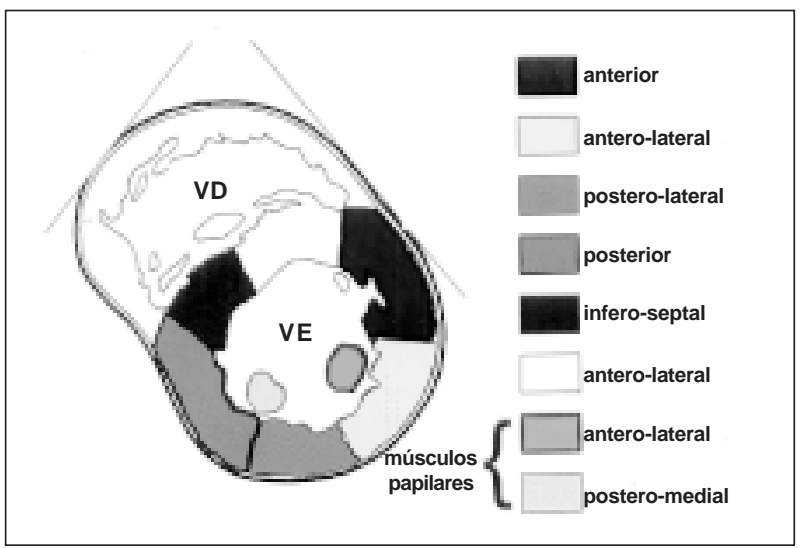

Fig. 1 - Schematic drawing of the division into segments of the parasternal short axis plane of the LV at the level of the papillary muscles. VD- right ventricle; VE- left ventricle; músculos papilares: papillary muscles 


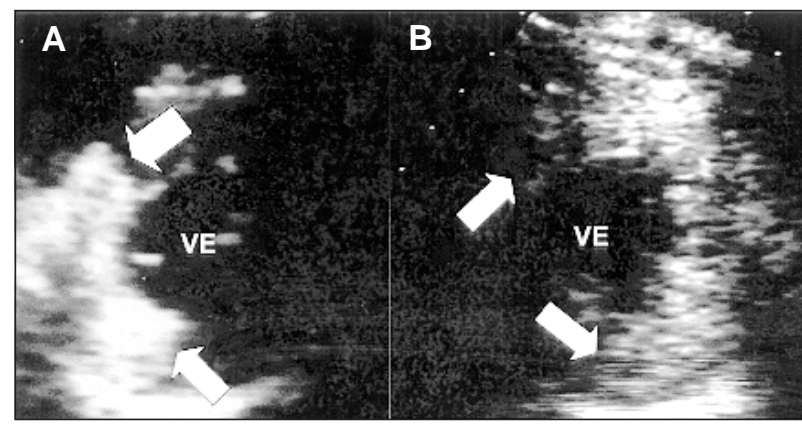

Fig. 2 - Perfused segment after injection in the right coronary artery (A) and in the left coronary artery (B). Limits can be well seen (arrows)

Figures 4 and 5 show how often segments are perfused, when related to the coronary arteries and when related to the dominant coronary artery, respectively.

In all 67 patients, antero-septal, anterior and anterolateral segments were perfused by the left coronary artery (LCA), independently of the dominant coronary artery.

The postero-lateral segment was perfused by the RCA in 65 of the 67 (97\%) patients- (in 7 with a dominant LCA and in 58 of the 60 patients who had the RCA as the dominant artery).

The posterior segment was perfused by the RCA in 53 (79\%) of the 67 patients but its perfusion was very dependent on the dominant coronary artery. When the RCA was dominant, this segment was perfused by the RCA in 51 $(85 \%)$ of the 60 patients. But when the LCA was dominant, the perfusion of the posterior segment was dependent on the LCA in $5(71 \%)$ of the 7 patients.

Similar data were observed for the infero-septal segment, which was perfused by the RCA in 51 (76\%) of the 67 patients, but also depended on the dominant artery. When RCA was dominant, this segment was perfused by the RCA in $49(82 \%)$ of the 60 patients. But, when the LCA was dominant, perfusion was dependent on the LCA in $5(71 \%)$ of the 7 patients, as occurred with the posterior segment.

Papillary muscles were well visualized in 59 patients. The anterolateral papillary muscle was perfused by the LCA in $58(98 \%)$ of the patients. In only one patient, who had a dominant RCA, was its perfusion dependent on the RCA. Conversely, posteomedial papillary muscle was perfused by

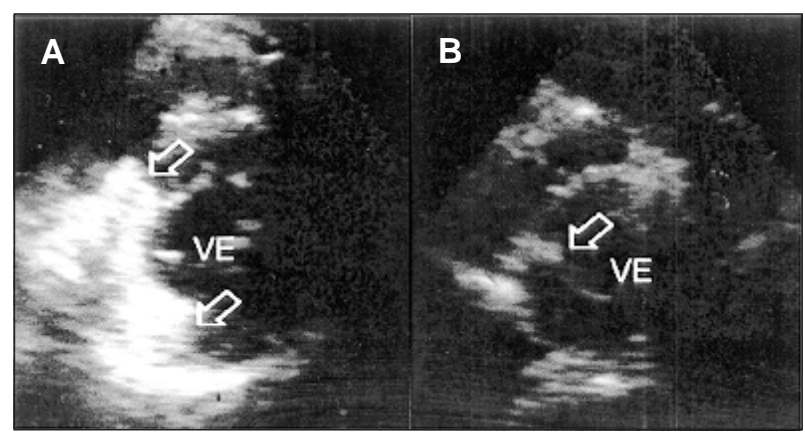

Fig. 3 - Perfusion bed size (arrows) in RCA dominance (A) and in the presence of a non-dominat RCA (B)

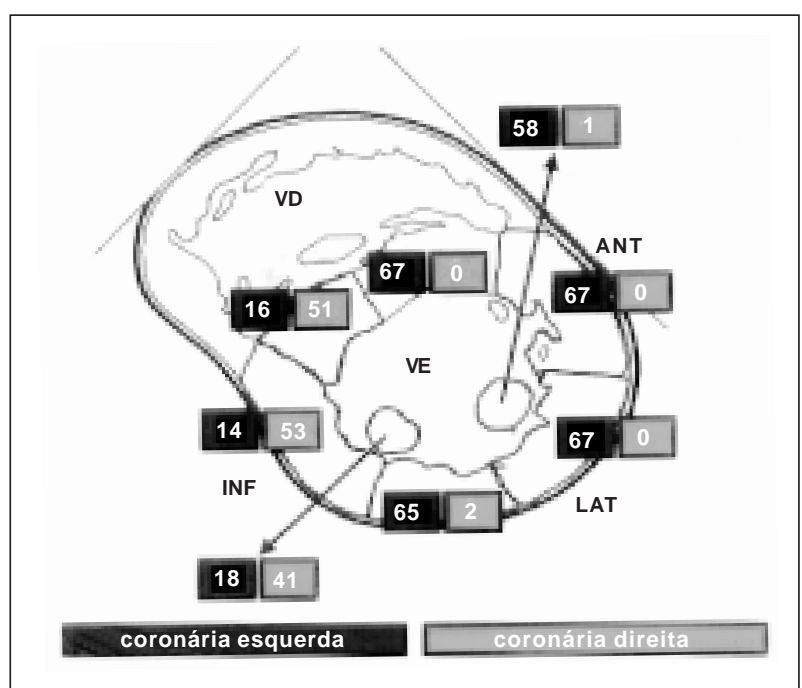

Fig. 4 - Schematic drawing of the left parasternal short axis plane of the LV at the level of the papillary muscles: frequency of perfusion and dominant coronary artery. Coronária esquerda: LCA; coronária direita: RCA.

the RCA in $41(69 \%)$ of the 59 patients but, as it happened in the posterior and the infero-septal segments, its perfusion was very dependent on the coronary dominance. When the RCA was dominant, this papillary muscle was perfused by the RCA in $40(77 \%)$ of the 52 patients. On the contrary, when LCA was dominant, its perfusion depended on the LCA in $6(86 \%)$ of the 7 patients.

No patient reported any symptoms when the sonicated solution was injected into the coronary artery. Small changes in the heart rate, in blood pressure and in the EKG were the same as those observed when the Pyelograph-76 was injected for routine coronary angiography

\section{Discussion}

In patients in whom coronary angiography showed no significant obstruction, when perfusion was investigated by selective injection of a Pyelograph-76 solution in each coronary artery, the qualitative pattern of contrast was

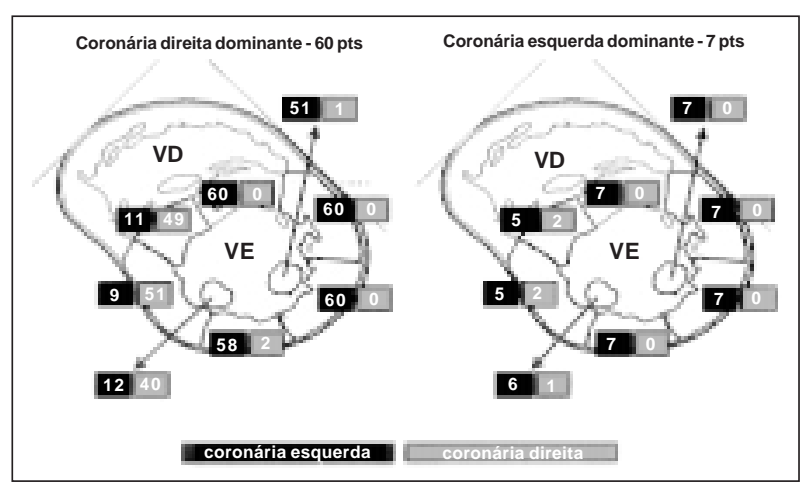

Fig. 5 - Schematic drawing of the left parasternal short axis plane of the LV at the level of the papillary muscles: frequency of perfusion by segments and dominant coronary artery. Coronária direita dominante- dominant RCA; coronária esquerda dominanted: dominant LCA; VD- right ventricle; VE- left ventricle. 
characterized by simultaneous opacification of all muscle layers, in all segments irrigated by the studied vessel.

Visual qualitative assessment of contrast appearance has not been the method of choice in this type of study. Most investigators ${ }^{9,27-29}$ have used video time-intensity quantitative assessment (VTI). These investigations performed measurements that analyzed contrast appearance ${ }^{9,27-29}$, such as peak contrast intensity and the time from the beginning to peak contrast. Others have analyzed washout time by analyzing the time duration and the area under the contrast curve ${ }^{27-29}$.

These studies were performed in laboratory animals where all conditions were controlled; animals could be compared pre- and postexperimental coronary artery occlusion, the same position for the intracoronary catheter was used and the same pressure and velocity were used for contrast injection. Therefore, validation and utility in the clinical scenarium has not been demonstrated yet. In this situation, there are no established values in the literature for standardization and comparison of a manual injection of the intracoronary contrast or for an established position for the catheter, which are almost impossible to obtain.

Contrast opacification distribution in the wall must be homogeneous for contrast echocardiography to become a useful clinical technique to investigate coronary perfusion. Contrary to that which was found by Griffin et al ${ }^{11}$, our study shows homogeneous myocardium contrast distribution in all segments perfused by the same artery and throughout the thickness of the wall. This difference may be explained by the fact that Griffin et al have used a manually agitated solution, not a sonicated one. Sonication of a solution for echocardiographic contrast use has been shown to produce a homogeneous distribution ${ }^{30}$.

Rovai et $\mathrm{al}^{31}$ found a nonuniform transmural contrast distribution, which was dependent on the phase of the cardiac cycle. When contrast was first seen in diastole, opacification was more pronounced in the subendocardial area; when it was first seen in systole, the subepicardial area was more evident. However, there was no observed delay for contrast appearance. These investigators used a video intensity quantitative assessment and do not mention if the difference could be visually detected.

Our study shows that patients with normal coronary arteries or without significant obstruction, have a large range of contrast peak intensity, in the time from beginning to peak intensity and in the washout time, even when these parameters are visually analyzed.

Transmural intersegmental distribution uniformity in contrast opacification can be qualitatively assessed and may be useful for evaluating myocardial perfusion. Clinical evaluation is independent of the manual drive force used during injection and from the intracoronary position of the catheter, since the analysis of the time of contrast appearance and the degree of opacification among wall segments and myocardial layers must be performed in the same injection.

Segment opacification of the LV walls defined in this study was dependent on the territory perfused by the studied coronary artery. Antero-septal, anterior and anterolateral segments were perfused by LCA in all cases, irrespective of the the dominant artery. The postero-lateral segment and the anterolateral papillary muscle, in the majority of cases, were also perfused by the LCA. Conversely, posterior and infero-septal segment and the posteromedial papillary muscle depended significantly on the coronary artery dominance, although, in most cases, they were perfused by a dominant RCA.

Echocardiographic myocardial contrast by an intracoronary injection of sonicated Pyelograph-76 was a safe procedure and no complications were observed. There were no significant hemodynamic side effects nor EKG abnormalities different from those observed during a usual coronary angiography.

Limitations - A simplified description of the coronary anatomy was used. Therefore, differences in the number and size of diagonal and obtuse marginal branches of the LCA, as well as the size of a nondominant RCA, were not taken into consideration.

Manual intracoronary contrast injection, performed by different investigators, is difficult to standardize. However, we don't think this is important for a qualitative analysis of the transmural and intersegmental distribution of the contrast.

The use of hyperosmolarity solutions, such as Pyelograph-76, can modify the coronary artery autoregulation system. Since all studies with contrast were performed after coronary angiography, it is possible that some autoregulation systems may have taken place and this may have interfered with the process.

In this study, in order to avoid further delay during the coronary angiography, only a short axis plane of the LV was obtained, but a more complete evaluation of myocardial perfusion should include other planes as well.

The study of myocardial perfusion using contrast echocardiography is a safe procedure. Intracoronary injection of $2 \mathrm{~mL}$ of a sonicated solution of Pyelograph-76 gives excellent myocardial opacification. This study shows the normal pattern of myocardial perfusion, evaluated by selective intracoronary injection of microbubbles in a significant number of patients. Visual, qualitative comparison of the time of appearance and of the contrast intensity in all layers and in different segments can be estimated by this technique. This can be of value in perfusion evaluation during a routine coronary angiography. 


\section{References}

1. DeMaria AN, Bommer WJ, Riggs K, et al. Echocardiographic visualization of myocardial perfusion by left heart and intracoronary injection of echo contrast agent. Circulation 1980; 62: III-143.

2. Kaul S, Pandian NG, Okada RD, Pohost GM, Weyman AE. Contrast echocardiography in acute myocardial ischemia. I. In vivo determination of total left ventricular "area at risk". J Am Coll Cardiol 1984; 4: 1272-82.

3. Kemper AJ, Force T, Perkins L, Gilfoil M, Parisi AF. In vivo prediction of the transmural extent of experimental acute myocardial infarction using contrast echocardiography. J Am Coll Cardiol 1986; 8: 143-9.

4. Kaul S, Kelly P, Oliner JD, Glasheen WP, Keller MW, Watson DD. Assessment of regional myocardial blood flow with myocardial contrast two-dimensional echocardiography. J Am Coll Cardiol 1989; 13: 468-82.

5. Kaul S, Pandian NG, Guerrero L, Gillam LD, Okada RD, Weyman AE. Effects of selectively altering collateral driving pressure on regional perfusion and function in occluded coronary bed in the dog. Circ Res 1987; 61: 77-85.

6. Kaul S, Glasheen WP, Oliner JD, Kelly P, Gascho JA. Relation between anterograde blood flow through a coronary artery and the size of the perfusion bed it supplies: experimental and clinical implications. J Am Coll Cardiol 1991; 17: $1403-13$.

7. Armstrong WF, Mueller TM, Kinney EL, Tickner EG, Dillon JC, Feigenbaum H Assessment of myocardial perfusion abnormalities with contrast-enhanced two-dimensional echocardiography. Circulation 1982; 66: 166-73.

8. Tei C, Sakamati T, Shah PM, et al. Myocardial contrast echocardiography: A reproducible technique of myocardial opacification for identifying regional perfusion deficits. Circulation 1983; 67: 585-93.

9. Kemper AJ, Force T, Kloner R, et al. Contrast echocardiography estimation of regional myocardial blood flow after acute coronary occlusion. Circulation 1985; 72: 1115-24.

10. Cheirif J, Desir RM, Bolli R, Mahmarian JJ. Relation of perfusion defects observed with myocardial contrast echocardiography to the severity of coronary stenosis: Correlation with Thallium-201 single-photon emission tomography. J Am Coll Cardiol 1992; 19: 1343-9.

11. Griffin B, Timmis AD, Sowton E. Contrast perfusion echocardiography: Distribution and reproducibility of myocardial contrast enhancement in coronary artery disease. Am J Cardiol 1987; 60: 538-43.

12. Kaul S, Jayaweera AR, Glasheen WP, Villanueva FS, Gutgesell HP, Spotnitz WD. Myocardial contrast echocardiography and the transmural distribution of flow: A critical appraisal during myocardial ischemia not associated with infarction. J Am Coll Cardiol 1992; 20: 1005-16.

13. Kaul S, Gillam LD, Weyman AE. Contrast echocardiography in acute myocardial ischemia, II: The effect of site of injection of contrast agent on the estimation of "area at risk" for necrosis after coronary occlusion. J Am Coll Cardiol 1985; 6: 825-30.

14. Kaul S, Glashenn W, Ruddy TD, Pandian NG, Weyman AE, Okada RD. The importance of defining left ventricular area at risk in-vivo during acute myocardial infarction: An experimental evaluation with myocardial contrast two-dimensional echocardiography. Circulation 1987; 75: 1249-60.

15. Ito H, Tomooka T, Sakai N, et al. Lack of myocardial perfusion immediately after successful thrombolysis. A predictor of poor recovery of left ventricular function in anterior myocardial infarction. Circulation 1992; 85: 1699-705.

16. Sabia PJ, Powers ER, Ragosta M, Sarembock IJ, Burwell LR, Kaul S. An association between collateral blood flow and myocardial viability in patients with recent myocardial infarction. N Engl J Med 1992; 327: 1825-31.

17. Ito $\mathrm{H}$, Tomooka $\mathrm{T}$, Sakai $\mathrm{N}$, et al. Time course of functional improvement in stunned myocardium in risk area in patients with reperfused anterior infarction. Circulation 1993; 87: 355-62.

18. Morcerf F. Identificação do miocárdio viável através da ecocardiografia de contraste. Rev Bras Eco 1997; 27: 30-5.

19. Lim Y, Nanto S, Lee J, et al. Coronary collaterals assessed with myocardial contrast echocardiography in healed myocardial infarction. Am J Cardiol 1990; 66: 556-61.

20. Widimsky P, Cornel JH, Ten Cate FJ. Evaluation of collateral blood flow by myocardial contrast enhanced echocardiography. Br Heart J 1988; 59: 20-2.

21. Sabia PJ, Powers ER, Jayaweera AR, Ragosta M, Kaul S. Functional significance of collateral blood flow in patients with recent acute myocardial infarction. A study using myocardial contrast echocardiography. Circulation 1992; 85: 2080-9.

22. Reisner AS, Ong LS, Lichtenberg GS, et al. Quantitative assessment of the immediate results of coronary angioplasty by myocardial contrast echocardiography. J Am Coll Cardiol 1989;13:852-9.

23. Grill HP, Brinker JA, Taube JC, et al. Contrast echocardiographic mapping of collateralized myocardium in humans before and after angioplasty. J Am Coll Cardiol 1990;16:1594-600.

24. Spotnitz WD, Kaul S. Intraoperative assessment of myocardial perfusion using contrast echocardiography. Echocardiography 1990; 7: 209-28.

25. Feinstein SB, Lang RM, Dick C, et al. Contrast echocardiography during coronary arteriography in humans: Perfusion and anatomic studies. J Am Coll Cardiol 1988; 11: 59-65.

26. Moore CA, Smucker ML, Kaul S. Myocardial contrast echocardiography in humans: I. Safety - A comparison with routine coronary arteriography. J Am Coll Cardiol 1986; 8: 1066-72.

27. Cheirif JB, Zoghbi WA, Bolli R, O'Neill PG, Hoyt BD, Quinones MA. Assessment of regional myocardial perfusion by contrast echocardiography. IIDetection of changes in transmural and subendocardial perfusion during dipyridamole-induced hyperemia in a model of critical coronary stenosis. J Am Coll Cardiol 1989; 14: 1555-65

28. Ten Cate FJ, Drury JK, Meerbaum S, et al. Myocardial contrast two-dimensional echocardiography: experimental examination at different coronary flow levels. J Am Coll Cardiol 1984; 3: 1219-26.

29. Kaul S. Animal studies: Part I. Echocardiography 1988; 5: 277-84.

30. Feinstein SB, Ten Cate FJ, Zwehl W, et al. Two-dimensional contrast echocardiography. I. In vitro development and quantitative analysis of echo contrast agents. J Am Coll Cardiol 1984; 3: 14-20.

31. Rovai D, L'Abbate A, Lombardi M, et al. Nonuniformity of the transmura distribution of coronary blood flow during the cardiac cycle. In vivo documentation by contrast echocardiography. Circulation 1989; 79: 179-87. 